\title{
Thermal diode in gas-filled nanogap with heterogeneous surfaces using nonequilibrium molecular dynamics simulation
}

T. Avanessian, and G. Hwang

Citation: Journal of Applied Physics 120, 165306 (2016); doi: 10.1063/1.4966599

View online: https://doi.org/10.1063/1.4966599

View Table of Contents: http://aip.scitation.org/toc/jap/120/16

Published by the American Institute of Physics

\section{Articles you may be interested in}

Nanoscale thermal transport. II. 2003-2012

Applied Physics Reviews 1, 011305 (2014); 10.1063/1.4832615

Thermal diodes, regulators, and switches: Physical mechanisms and potential applications

Applied Physics Reviews 4, 041304 (2017); 10.1063/1.5001072

Improvement of heat transfer efficiency at solid-gas interfaces by self-assembled monolayers

Applied Physics Letters 102, 061907 (2013); 10.1063/1.4792530

Molecular dynamics simulation of effective thermal conductivity of vapor-filled nanogap and nanocavity

Journal of Applied Physics 106, 024317 (2009); 10.1063/1.3186043

A simple nonequilibrium molecular dynamics method for calculating the thermal conductivity

The Journal of Chemical Physics 106, 6082 (1997); 10.1063/1.473271

Thermal rectification in silicon by a graded distribution of defects

Journal of Applied Physics 119, 215102 (2016); 10.1063/1.4953142

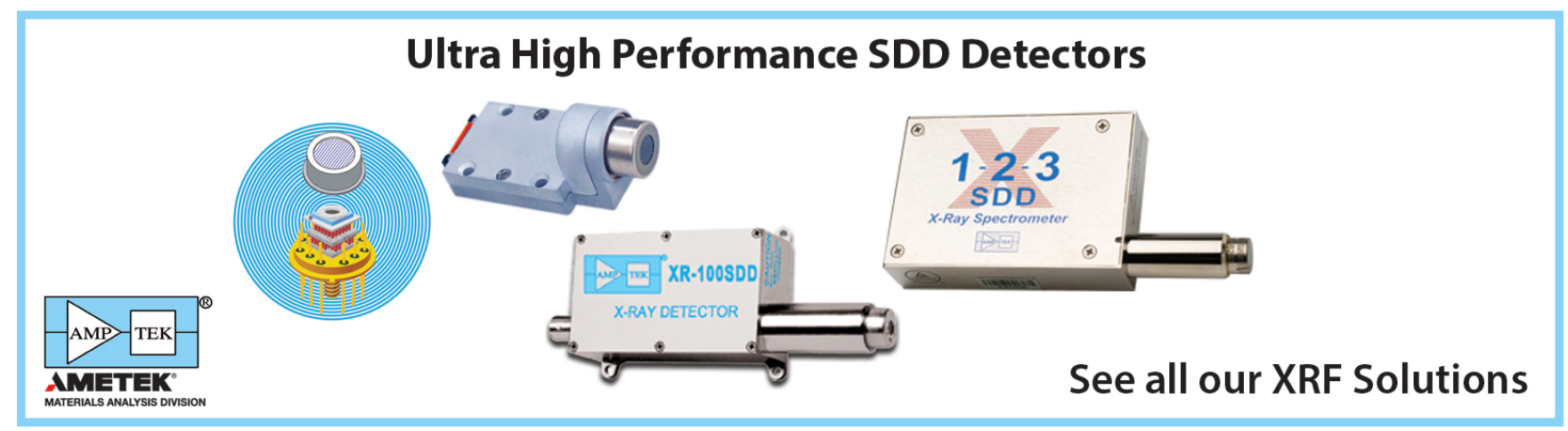




\title{
Thermal diode in gas-filled nanogap with heterogeneous surfaces using nonequilibrium molecular dynamics simulation
}

\author{
T. Avanessian and G. Hwang ${ }^{\text {a) }}$ \\ Department of Mechanical Engineering, Wichita State University, Wichita, Kansas 67260, USA
}

(Received 20 September 2016; accepted 16 October 2016; published online 28 October 2016)

\begin{abstract}
A thermal diode serves as a basic building block to design advanced thermal management systems in energy-saving applications. However, the main challenges of existing thermal diodes are poor steadystate performance, slow transient response, and/or extremely difficult manufacturing. In this study, the thermal diode is examined by employing an argon gas-filled nanogap with heterogeneous surfaces in the Knudsen regime, using nonequilibrium molecular dynamics simulation. The asymmetric gas pressure and thermal accommodation coefficients changes are found due to asymmetric adsorptions onto the heterogeneous nanogap with respect to the different temperature gradient directions, and these in turn result in the thermal diode. The maximum degree of diode (or rectification) is $R_{\max } \sim 7$, at the effective gas-solid interaction ratio between the two surfaces of $\varepsilon^{*}=0.75$. This work could pave the way to designing advanced thermal management systems such as thermal switches (transistors). Published by AIP Publishing. [http://dx.doi.org/10.1063/1.4966599]
\end{abstract}

\section{INTRODUCTION}

A thermal diode (or rectifier) transfers heat only in the favorable direction, which is strongly needed to recycle the waste heat energy, ${ }^{1-3}$ efficiently use the building energy, ${ }^{4}$ and completely open new energy-saving applications, i.e., thermal computers. ${ }^{5-9}$ However, the main challenges of such systems lie in the extremely difficult manufacturing, poor steady-state efficiency, slow transient response, limited operation conditions, and/or required expensive external thermal control systems. ${ }^{6,10-14}$ The degree of the thermal diode (or rectification), $R$, is defined as

$$
R=\frac{\left|q_{+}\right|-\left|q_{-}\right|}{\left|q_{-}\right|}
$$

where $\left|q_{+}\right|$and $\left|q_{-}\right|$are heat fluxes in the favorable and unfavorable directions, respectively, and the thermal diode requires that $R>0$.

Various designs employing nonlinear heat transfer characteristics, both experimentally and theoretically, have been explored. ${ }^{8,13,14}$ The first thermal diode has been experimentally demonstrated in the solid state, i.e., $\mathrm{Cu} / \mathrm{CuO}$ interface, $R_{\max } \sim 1.7$. $^{15}$ The recent emergence of nanotechnology has led to experiments involving the use of asymmetric carbon nanotube, $R_{\text {max }} \sim 0.07 ;^{6}$ asymmetric graphene oxide, $R_{\max } \sim 0.25 ;{ }^{16}$ electrochemically tuned thermal diode material, $R_{\max } \sim 0.5{ }^{17}$ and asymmetric radiative thermal transport, $R_{\max } \sim 0.11 .^{9}$ To improve the relatively low $R$, liquid-based systems have been employed using nonlinear convection in heat pipes, $R_{\text {max }} \sim 200^{18}$ and $1000^{19}$ or heterogeneous water wettings, $R_{\text {max }} \sim 100^{20}$ and $0.46 .^{21}$ However, liquid-based systems result in a relatively slow transient response $\sim 10 \mathrm{~min}$, which may limit their performance in some applications. Numerous theoretical frameworks for efficient thermal diode mechanisms have been explored in the literature, ${ }^{2,3,7,13,14,22-51}$ but those

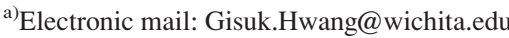

have not been demonstrated in experiments, due to the necessary expensive or challenging, atomic-level manufacturing technologies. These mechanisms also include nonlinear quantum systems, ${ }^{2,25-31}$ which should be a long-term approach for the experiments. Further experimental improvement in steadystate and transient performance requires an innovative approach using a viable theoretical framework for the experimental realization.

\section{ADSORPTION-CONTROLLED THERMAL DIODE}

In this study, a thermal diode is examined using a gasfilled nanogap with heterogeneous gas-solid surface interactions as shown in Fig. 1. This aims at developing a new class of thermal diode mechanism via nonlinear gas conduction for efficient steady-state performance and fast transient response.

The thermal conductivity in the gas-filled nanogap (Knudsen regime) is well understood using a kinetic theory. ${ }^{52,53}$ In Knudsen regime $\left(\mathrm{Kn}_{L}=\lambda_{f} / L_{z}\right)$, where the characteristic length of the nanogap, $L_{z}$, is similar to the mean free path, $\lambda_{f}$, the effective thermal conductivity across the gasfilled nanogap is related to the thermal accommodation coefficients (TACs) of both surfaces and the ballistic thermal transport through the gas molecules. In $0.1<\mathrm{Kn}_{L}<10$ (transition regime), the effective thermal conductivity of gas confined in the nanostructure is given as 52,53

$$
\left\langle k_{f, t}\right\rangle=\left\langle k_{f, f m}\right\rangle\left(1+\frac{4}{15} \frac{1}{\mathrm{Kn}_{L}} \frac{a_{T, 1} a_{T, 2}}{a_{T, 1}+a_{T, 2}-a_{T, 1} a_{T, 2}}\right)^{-1},
$$

where $\left\langle k_{f, f m}\right\rangle$ is the effective thermal conductivity in free molecular regime $\left(\mathrm{Kn}_{L}>10\right)$, and $a_{T, 1}$ and $a_{T, 2}$ are the TACs at surfaces 1 and 2, respectively. TAC is defined as

$$
a_{T}=\frac{\left\langle q_{f, z}\right\rangle-\left\langle q_{f, z}\right\rangle^{\prime}}{\left\langle q_{f, z}\right\rangle-\left\langle q_{f, z}\left(T_{s}\right)\right\rangle},
$$


(a) $T_{1}>T_{2},\left\langle\boldsymbol{q}_{+}\right\rangle>>0$

(b) $T_{1}<T_{2},\left\langle\boldsymbol{q}_{-}\right\rangle \sim 0$

Weak Surface Force

(Artificial Surface)

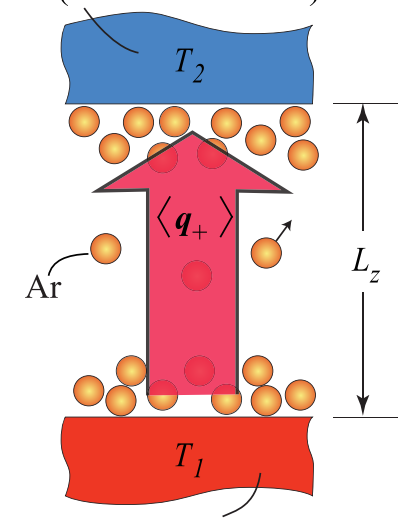

Strong Surface Force (Pt)

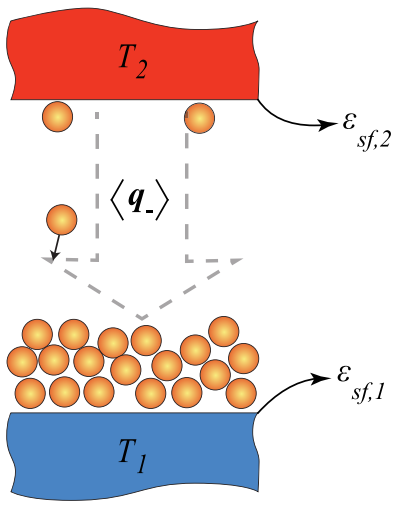

FIG. 1. A schematic of the adsorption-controlled thermal diode using a gasfilled nanogap. (a) $\left\langle q_{+}\right\rangle \gg 0$ for $T_{1}>T_{2}$ and (b) $\left\langle q_{-}\right\rangle \sim 0$ for $T_{1}<T_{2}$ due to the adsorption-controlled asymmetric isotherm and thermal accommodation coefficient (TAC). The surface temperatures, $T_{1}$ and $T_{2}$, heat fluxes, $\left\langle q_{+}\right\rangle$ and $\left\langle q_{-}\right\rangle$, gas-solid interactions, $\varepsilon_{s f, 1}$ and $\varepsilon_{s f, 2}$, nanogap size, $L_{z}$, and the adsorbed particles are also shown.

where $\left\langle q_{f, z}\right\rangle$ and $\left\langle q_{f, z}\right\rangle^{\prime}$ are the heat flux of the impinging and reflecting gas molecules to/from the solid surface, respectively, and $\left\langle q_{f, z}\left(T_{s}\right)\right\rangle$ is the heat flux between the solid and gas molecules.

The demonstration of the thermal diode in the gas-filled nanogap requires either asymmetric ballistic thermal transport via gas atoms, and/or controlled interfacial thermal transport via TACs with respect to a temperature gradient direction. To control such thermal transport, an Ar gas-filled nanogap with heterogeneous materials is employed having Pt for the lower surface $\left(T_{1}\right)$ and an artificial material for the upper surface $\left(T_{2}\right)$ that has a weaker gas-solid interaction compared to the lower surface. At an equilibrium state, i.e., $T_{1}=T_{2}$, gas atoms are more attractive to the solid surface due to the stronger gas-solid interaction than fluid-fluid interaction, so called adsorption, but the amount of adsorption onto the Pt surface is larger than that of the weaker gas-solid interacting surface due to smaller attraction, i.e., heterogeneous adsorption. At a nonequilibrium state, $T_{1} \neq T_{2}$, the amount of the adsorption is different from that of the equilibrium state, since the local thermal equilibrium of each surface changes from the different surface temperatures. In fact, the amount of adsorption is determined by the combination of the gas-solid interaction energy and the surface temperature; the stronger the surface energy, the greater is the amount of the adsorption. ${ }^{54}$ When the temperature gradient is upward $\left(T_{1}>T_{2}\right)$, both surfaces have moderate number of adsorbed particles, because the higher temperature of the $\mathrm{Pt}$ surface (strong interaction) desorbs Ar atoms, while the lower temperature of the upper surface (weak interaction) adsorbs them. This moderate adsorption on both surfaces leads to large amounts of TAC on both surfaces, resulting in a significant heat transfer across the interfaces. On the other hand, when the temperature gradient is in the opposite direction, the high temperature with small gas-solid interaction results in a poor adsorption on surface 2 , which leads to a low TAC, whereas the lower temperature with large gassolid interaction causes a significant adsorption on surface 1 and results in high TAC. The poor interfacial heat transfer across surface 2 in turn results in a poor overall heat transfer, and this leads to the asymmetric heat transfer, i.e., thermal diode (or rectification). A key heat transfer control mechanism is to control TAC, which requires a nanogap size of the same order of the gas mean free path.

\section{METHODOLOGY}

The thermal diode is calculated using nonequilibrium molecular dynamics simulation (NEMDS). A brief description of the non-equilibrium molecular dynamics simulations is given here and details could be found in the supplementary material including the validation of NEMDS (Fig. S1). 576 argon atoms are filled in a nanogap of $L_{z}=20 \mathrm{~nm}$ with $L_{x}=8.864 \mathrm{~nm}$ and $L_{y}=7.676 \mathrm{~nm}$. Periodic boundary conditions are used for $x$ and $y$ directions, while non-periodic boundary condition is used for $z$ direction. Note that the thermal diode is achieved by the control of thermal accommodation coefficient, which requires the nanostructure size to have the same order of the mean free path of the gas. Here, $L_{z}=20 \mathrm{~nm}$ for the gas pressure of 10 to $800 \mathrm{kPa}$ and the temperature of 80 to $130 \mathrm{~K}$.

A temperature difference of $\Delta T=T_{1}-T_{2}= \pm 20 \mathrm{~K}$ is used across the nanogap to create the temperature gradient, and the resulting ensemble-averaged heat flux across the gasfilled nanogap is calculated using the given relation ${ }^{5,56}$

$$
\langle q\rangle=\frac{1}{V}\left[\sum_{i=1}^{N} \frac{1}{2} m_{i} u_{i}^{2} u_{i, z}+\sum_{i=1}^{N} \sum_{j>1}^{N} \varphi_{i j} u_{i, z}-\sum_{i=1}^{N} \sum_{j>1}^{N}\left(\mathbf{r}_{\mathbf{i j}} \cdot \mathbf{F}_{\mathbf{i}}\right) u_{i, z}\right],
$$

where $V$ is the volume, $m_{i}$ is the mass of the argon particle, $u_{i}$ is the particle velocity, $\varphi_{i}$ is the potential energy, $\mathbf{r}_{\mathbf{i j}}$ is the displacement separation vector between $i$ th and $j$ th atoms, and $\mathbf{F}_{\mathbf{i}}$ is the interactive force vector. The key design parameter for the thermal diode is the asymmetric gas-solid interaction in surfaces 1 and 2. Here, the degree of the asymmetric interaction parameter is given as $\varepsilon^{*}=\varepsilon_{s f, 2} / \varepsilon_{s f, 1}$. We used $\varepsilon_{s f, I}=0.1573 \mathrm{kcal} / \mathrm{mol}$ with $\varepsilon^{*}=0.75$ and 0.5 to have heterogeneous surface.

\section{RESULTS AND DISCUSSION}

The predicted heat fluxes [Eq. (4)] are shown in Fig. 2 as a function of the average nanogap temperature, i.e., $80 \leq\langle T\rangle \leq 130$, for $\varepsilon^{*}=1.0,0.75$, and 0.5 .

Note that the gas temperature associated with the average nanogap temperature is found in Section B of the supplementary material. For $\varepsilon^{*}=1.0$, the heat flux increases with increasing the average nanogap temperature. A higher surface temperature desorbs the Ar particles (under the fixed number of Ar atoms), resulting in a higher gas pressure and heat flux. In fact, although the high surface temperature results in a lower TAC (lower heat flux), the heat flux enhancement by the increased pressure outweighs the heat 


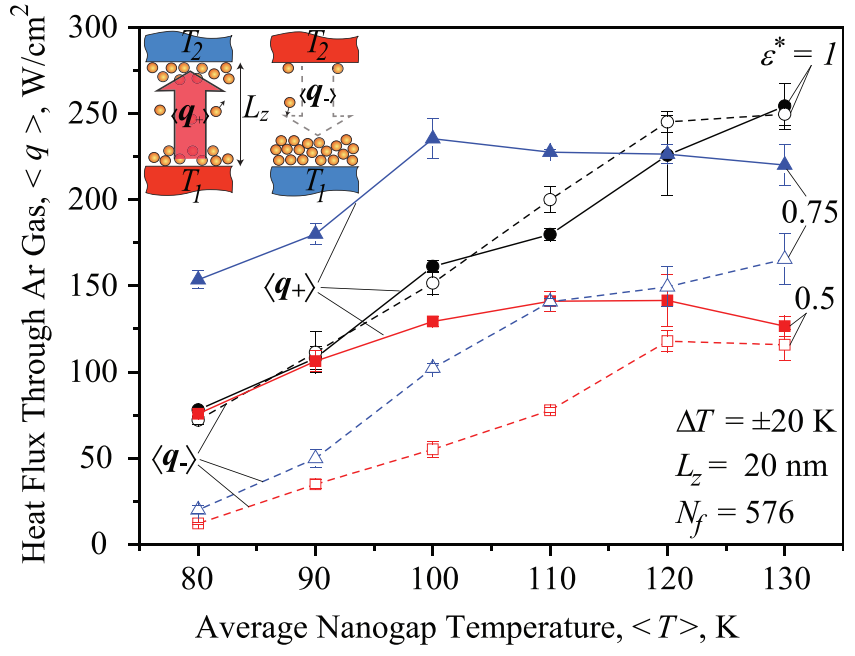

FIG. 2. Variations of heat flux through the nanogap as a function of average surface temperature both in $\left\langle q_{+}\right\rangle$(solid symbols) and $\left\langle q_{-}\right\rangle$(empty symbols) directions, for $\varepsilon^{*}=1.0,0.75$, and 0.5 . The error bars and insets for the simulation setup are also shown.

flux decrease by the lower TAC. No significant difference between $\left\langle q_{+}\right\rangle$and $\left\langle q_{-}\right\rangle$is found due to the symmetric heat transfer for both temperature gradient directions, i.e., no diode effect, $R \sim 0$. However, for $\varepsilon^{*}=0.75$ and 0.5 (asymmetric surface interaction), the heat flux when $T_{1}>T_{2}$ is much higher than when $T_{1}<T_{2}$. This asymmetric heat flux is more pronounced in low temperatures, due to significant asymmetric adsorptions onto the nanogap surfaces and resulting gas pressure variation when the direction of temperature gradient changes. Note that there are peaks in $\left\langle q_{+}\right\rangle$ at $100 \mathrm{~K}$ and $110 \mathrm{~K}$ for $\varepsilon^{*}=0.75$ and 0.5 , respectively. These deflection points are related to the combined effect of gas pressure-TACs on the heat flux; for lower temperatures, the heat flux increases by increasing the gas pressure without decreasing TACs, whereas for higher temperatures, the heat flux begins to decrease due to the poor TACs.

Using the obtained heat fluxes, the variations of $R$ [Eq. (1)] for $\varepsilon^{*}=1.0,0.75$, and 0.5 are calculated as shown in Fig. 3. For $\varepsilon^{*}=1.0, R \sim 0$ due to the symmetric heat flux through the homogeneous nanogap. For $\varepsilon^{*}=0.75$ and 0.5 , the maximum degrees of the rectification are $R_{\max }=6.7$ and 5.6 at $T=80 \mathrm{~K}$, respectively, due to the adsorptioncontrolled, gas pressure and TAC. The thermal diode effect decreases down to $R \sim 0.1$, at high temperatures, i.e., $T=130 \mathrm{~K}$, due to no significant adsorption-controlled gas pressure and TAC, i.e., almost identical nanogap pressure for heat flux in positive and negative directions.

To further understand the asymmetric heat flux, the number density of adsorbed Ar atoms near the surfaces, $n_{f}$, is calculated as a function of surface temperature, and the results are shown in Fig. 4. The number density of the gas region is shown in Fig. S2 of the supplementary material.

In Fig. 4(a), for $\varepsilon^{*}=1.0$ (homogeneous surfaces), the number density of adsorbed particles on the cold temperature surface is higher than that on the hot surface, but it is lower than the liquid Ar. As the surface temperature increases, the number density on the cold surface decreases due to the desorption (or higher kinetic energy of Ar particles), while

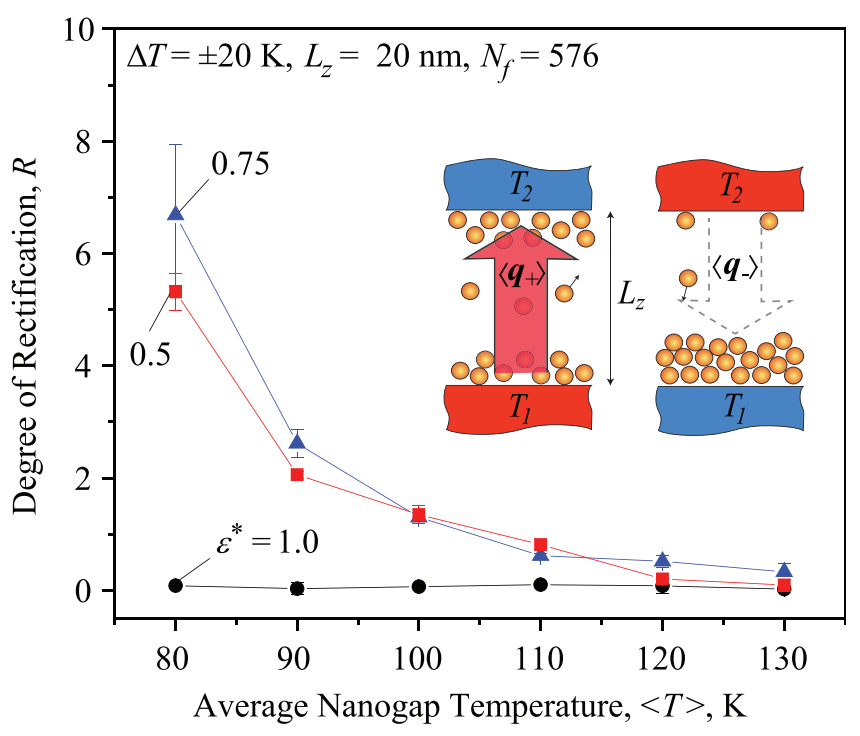

FIG. 3. Variation of the degree of thermal diode (rectification) using the adsorption-controlled heat flux as a function of average nanogap temperature, for $\varepsilon^{*}=1.0,0.75$, and 0.5 [Eq. (1)]. Some error bars are smaller than the size of their corresponding symbols.

the number density on the hot surface increases due to the adsorption by increased gas pressure. More importantly, when the temperature gradient direction changes, the same number densities are determined near both cold and hot surfaces, i.e., no thermal diode effect. In the case of $\varepsilon^{*}=0.75$, for $\left\langle q_{+}\right\rangle$, both surfaces have similar number densities, decreasing with increasing the surface temperature, whereas for $\left\langle q_{-}\right\rangle$, the number densities of both surfaces are significantly different due to the heterogeneous gas-solid interactions [Fig. 4(a)]. This in turn results in asymmetric heat flux, i.e., thermal diode. In the case of $\varepsilon^{*}=0.5$, for $\left\langle q_{+}\right\rangle$, the number density near the cold surface is considerably higher than that near the hot surface, i.e., the difference between them is greater than that for $\varepsilon^{*}=0.75$ due to the large surface interaction heterogeneity, and this difference is more pronounced at low temperatures.

The origin of the thermal diode effect is further articulated by calculating the asymmetric TAC on the heterogeneous gas-solid interactions, $\varepsilon^{*}=0.75$ and 0.5 , as shown in Fig. 5. For $\varepsilon^{*}=1.0$, the TAC is high, $a_{T} \sim 0.9$, at low surface temperatures due to the significant adsorption and it decreases down to $a_{T} \sim 0.5$ as the surface temperature increases up to $T=140 \mathrm{~K}$. The predicted TACs reasonably agree with that of the previous studies. ${ }^{57-59}$ Moreover, the TAC does not change when reversing the temperature gradient direction. However, for $\varepsilon^{*}=0.75$ [Fig. 5(a)], in $\left\langle q_{+}\right\rangle$, the TAC for the strong surface is significantly higher than that for the weak surface, and TACs for both surfaces decrease with increasing surface temperatures. In $\left\langle q_{-}\right\rangle$, the TAC on the strong surface is similar to that for $\left\langle q_{+}\right\rangle$, whereas the TAC on the weak surface is lower than that for $\left\langle q_{+}\right\rangle$, especially at low temperatures. This asymmetric TAC causes the thermal diode. For $\varepsilon^{*}=0.5$ [Fig. 5(b)], the difference between the TACs in $\left\langle q_{+}\right\rangle$ and $\left\langle q_{-}\right\rangle$is much larger than the case for $\varepsilon^{*}=0.75$, but the TACs are symmetric between $\left\langle q_{+}\right\rangle$and $\left\langle q_{-}\right\rangle$. Even though the TACs are symmetric, we observe the significant thermal diode effect as shown in Fig. 3. 
(a)

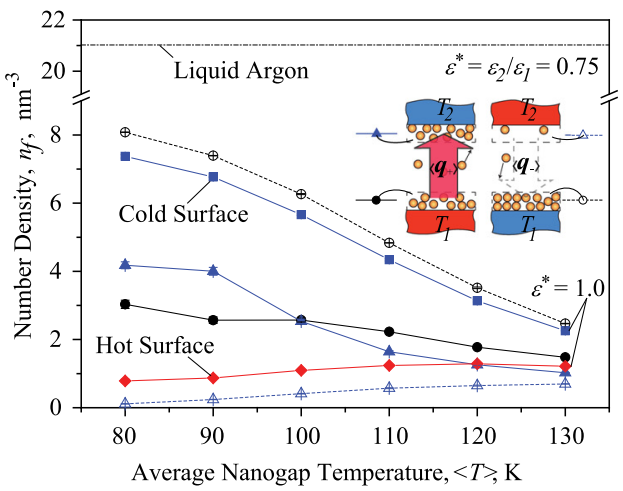

(a)

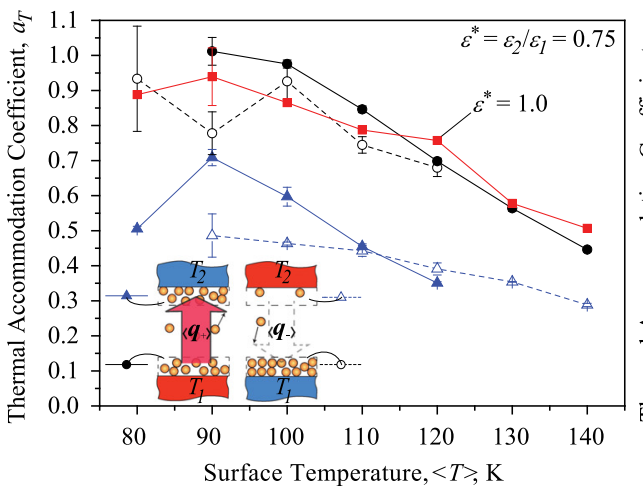

(b)

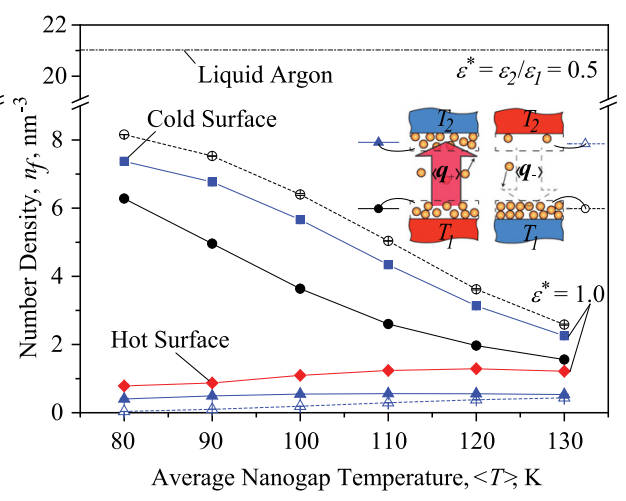

(b)

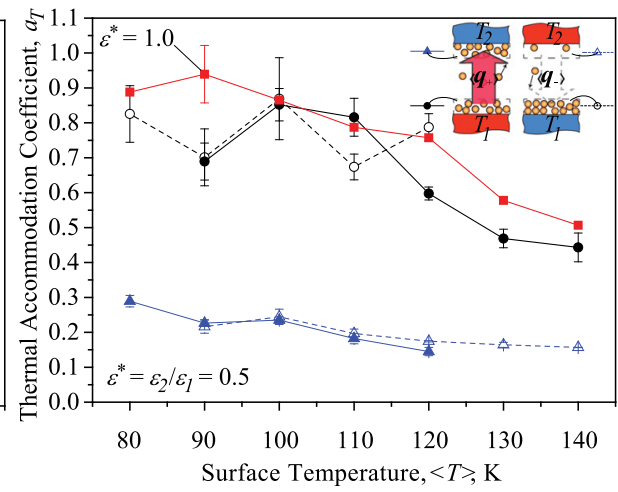

FIG. 4. The number density of adsorbed particles on the lower and upper surfaces for $\left\langle q_{+}\right\rangle$(solid symbols) and $\left\langle q_{-}\right\rangle$(empty symbols), (a) $\varepsilon^{*}$ $=0.75$ and (b) $\varepsilon^{*}=0.5$. The insets with the adsorbed Ar particles are also shown. Error bars are shown, but those are smaller than the size of their symbols. The number density of liquid $\mathrm{Ar}$ is also shown.
To further elucidate the origin of the thermal diode effect, the gas pressure of the nanogap is calculated as shown in Fig. 6 as a function of average nanogap temperature. For $\varepsilon^{*}=1.0$, the gas pressure is symmetric; however, for $\varepsilon^{*}=0.5$, the gas pressure for $\left\langle q_{+}\right\rangle$is much higher than that for $\left\langle q_{-}\right\rangle$due to large desorption from the strong surface for $\left\langle q_{+}\right\rangle$and significant adsorption onto the strong surface for $\left\langle q_{-}\right\rangle$.

This indicates the dominant source of the thermal diode effect for reversed temperature gradient direction where TACs are symmetric, as shown in Fig. 5(b). For $\varepsilon^{*}=0.75$, this gas pressure change is less pronounced compared to $\varepsilon^{*}=0.5$, i.e., by a factor of $2\left(\left\langle p_{g,+}\right\rangle /\left\langle p_{g,-}\right\rangle \sim 9\right.$ for $\varepsilon^{*}=0.5$

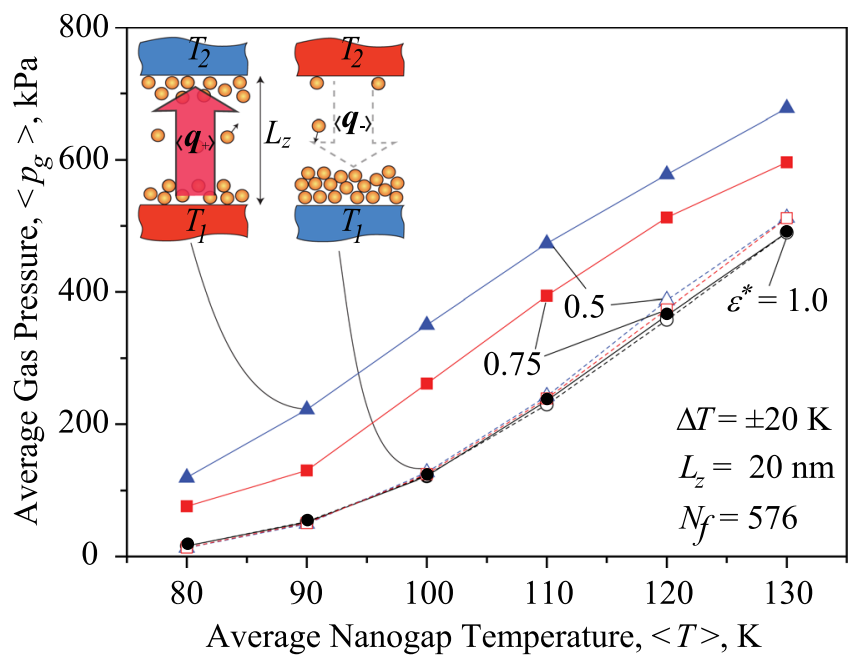

FIG. 6. Average pressure within the nanogap far from the effect of gassurface interaction both in $\left\langle q_{+}\right\rangle$(solid symbols) and $\left\langle q_{-}\right\rangle$(empty symbols) directions for $\varepsilon^{*}=1.0,0.75$, and 0.5 . Error bars are smaller than symbol size. and $\left\langle p_{g,+}\right\rangle /\left\langle p_{g,-}\right\rangle \sim 5.5$ for $\varepsilon^{*}=0.75$ at $\langle T\rangle=80 \mathrm{~K}$ ), showing that the change in gas pressure combined with the asymmetric TAC causes the thermal diode.

\section{CONCLUSIONS}

In this study, the adsorption-controlled thermal diode is examined using the Ar-gas-filled heterogeneous nanogap. The thermal diode effect is caused by the asymmetric gas pressure combined with TACs under different strengths of solid-gas interaction, showing the maximum degree of rectification, $R_{\max } \sim 7$ for $\varepsilon^{*}=0.75$ at $T=80 \mathrm{~K}$. For $\varepsilon^{*}=0.75$, the thermal diode is originated by the combination of the adsorption-controlled asymmetric gas pressure and TACs, while for $\varepsilon^{*}=0.5$, it is dominantly controlled by the asymmetric gas pressure. The obtained results for the Ar-filled Pt-based nanogap with the tailored materials provide a design guideline for the thermal diode using real materials. $\varepsilon^{*}=0.75$ and 0.5 can be designed using $\mathrm{Pt} / \mathrm{Ni}$ and $\mathrm{Pt} / \mathrm{Al}$ as strong/weak surfaces based on the LJ potential in literature, respectively. ${ }^{60}$ The developed thermal diode can serve as a basic building block to design advanced thermal systems such as thermal switches and thermal computing systems.

\section{SUPPLEMENTARY MATERIAL}

See supplementary material for additional information on the simulation.

\section{ACKNOWLEDGMENTS}

This study is based upon the work supported by the National Science Foundation under Award No. EPS-0903806 
and matching support from the State of Kansas through the Kansas Board of Regents. This work was also partially supported by the start-up fund from the College of Engineering, Wichita State University. This work also used the Extreme Science and Engineering Discovery Environment (XSEDE), which was supported by National Science Foundation Grant No. ACI-1053575.

${ }^{1}$ A. A. Mohamad, Sol. Energy 61, 211 (1997).

${ }^{2}$ D. M. T. Kuo and Y.-C. Chang, Phys. Rev. B 81, 205321 (2010).

${ }^{3}$ N. A. Roberts and D. G. Walker, J. Heat Transfer 133, 092401 (2011).

${ }^{4}$ S. Varga, A. C. Oliveira, and C. F. Afonso, Energy Build. 34, 227 (2002).

${ }^{5}$ L. Wang and B. Li, Phys. Rev. Lett. 99, 177208 (2007).

${ }^{6}$ C. W. Chang, D. Okawa, A. Majumdar, and A. Zettl, Science 314, 1121 (2006).

${ }^{7}$ G. Casati, Nat. Nanotechnol. 2, 23 (2007).

${ }^{8}$ N. Li, J. Ren, L. Wang, G. Zhang, P. Hänggi, and B. Li, Rev. Mod. Phys. 84, 1045 (2012).

${ }^{9}$ Z. Chen, C. Wong, S. Lubner, S. Yee, J. Miller, W. Jang, C. Hardin, A. Fong, J. E. Garay, and C. Dames, Nat. Commun. 5, 5446 (2014).

${ }^{10}$ R. P. Bywaters and R. A. Griffin, Cryogenics 13, 344 (1973).

${ }^{11}$ I. Catarino, G. Bonfait, and L. Duband, Cryogenics 48, 17 (2008).

${ }^{12}$ C. Dames, J. Heat Transfer 131, 061301 (2009).

${ }^{13}$ N. A. Roberts and D. G. Walker, Int. J. Therm. Sci. 50, 648 (2011).

${ }^{14} \mathrm{G}$. Hwang and T. Avanessian, in Multiscale Thermal Transport in Energy Systems, edited by Y.-L. He and Y. Zhang (Nova Science Publishers, Inc., 2016).

${ }^{15}$ C. Starr, J. Appl. Phys. 7, 15 (1936).

${ }^{16}$ H. Tian, D. Xie, Y. Yang, T.-L. Ren, G. Zhang, Y.-F. Wang, C.-J. Zhou, P.-G. Peng, L.-G. Wang, and L.-T. Liu, Sci. Rep. 2, 523 (2012).

${ }^{17}$ J. Cho, M. D. Losego, H. G. Zhang, H. Kim, J. Zuo, I. Petrov, D. G. Cahill, and P. V. Braun, Nat. Commun. 5, 4035 (2014).

${ }^{18}$ M. Groll, W. D. Munze, W. Supper, and C. J. Savage, J. Spacecr. Rockets 16, 195 (1979).

${ }^{19}$ J. Alario, "Cryogenic thermal diode heat pipes," Report No. NASA CR$152,268,1979$.

${ }^{20}$ J. B. Boreyko, Y. Zhao, and C.-H. Chen, Appl. Phys. Lett. 99, 234105 (2011).

${ }^{21}$ T. Hirayanagi, T. Tsukamoto, M. Esashi, and S. Tanaka, J. Phys. Conf. Ser. 476, 012019 (2013).

${ }^{22}$ M. Hu, J. V. Goicochea, B. Michel, and D. Poulikakos, Appl. Phys. Lett. 95, 151903 (2009).

${ }^{23}$ S. Murad and I. K. Puri, Appl. Phys. Lett. 100, 121901 (2012).

${ }^{24}$ F. Yuan and L. Xin-Gang, Heat Transport Through a Solid-Liquid Interface System and its Thermal Rectification Phenomena by Molecular Dynamics Simulations, Chicago, 2014.
${ }^{25}$ J.-P. Eckmann and C. Mejía-Monasterio, Phys. Rev. Lett. 97, 094301 (2006).

${ }^{26}$ D. Segal, Phys. Rev. Lett. 100, 105901 (2008).

${ }^{27}$ L.-A. Wu and D. Segal, Phys. Rev. Lett. 102, 095503 (2009).

${ }^{28}$ X.-O. Chen, B. Dong, and X.-L. Lei, Chin. Phys. Lett. 25, 3032 (2008).

${ }^{29}$ R. Scheibner, M. König, D. Reuter, A. D. Wieck, C. Gould, H. Buhmann, and L. W. Molenkamp, New J. Phys. 10, 083016 (2008).

${ }^{30}$ T. Ruokola, T. Ojanen, and A.-P. Jauho, Phys. Rev. B 79, 144306 (2009).

${ }^{31}$ T. Ojanen, Phys. Rev. B 80, 180301 (2009).

${ }^{32}$ B. Li, L. Wang, and G. Casati, Phys. Rev. Lett. 93, 184301 (2004).

${ }^{33}$ M. Terraneo, M. Peyrard, and G. Casati, Phys. Rev. Lett. 88, 094302 (2002).

${ }^{34}$ J. Hu, X. Ruan, and Y. P. Chen, Nano Lett. 9, 2730 (2009).

${ }^{35}$ Y. Wang, S. Chen, and X. Ruan, Appl. Phys. Lett. 100, 163101 (2012).

${ }^{36}$ Y. Wang, A. Vallabhaneni, J. Hu, B. Qiu, Y. P. Chen, and X. Ruan, Nano Lett. 14, 592 (2014).

${ }^{37}$ M. Alaghemandi, F. Leroy, E. Algaer, M. C. Böhm, and F. Müller-Plathe, Nanotechnology 21, 075704 (2010).

${ }^{38}$ S. Pal and I. K. Puri, Nanotechnology 25, 345401 (2014).

${ }^{39}$ N. Yang, G. Zhang, and B. Li, Appl. Phys. Lett. 93, 243111 (2008).

${ }^{40}$ G. Wu and B. Li, Phys. Rev. B 76, 085424 (2007).

${ }^{41}$ N. Yang, G. Zhang, and B. Li, Appl. Phys. Lett. 95, 033107 (2009).

${ }^{42}$ G. Balasubramanian, I. K. Puri, M. C. Bohm, and F. Leroy, Nanoscale 3, 3714 (2011)

${ }^{43}$ L. P. Wang and Z. M. Zhang, Nanoscale Microscale Thermophys. Eng. 17, 337 (2013).

${ }^{44}$ L. Zhu, C. R. Otey, and S. Fan, Phys. Rev. B 88, 184301 (2013).

${ }^{45}$ C. R. Otey, W. T. Lau, and S. Fan, Phys. Rev. Lett. 104, 154301 (2010).

${ }^{46}$ H. Iizuka and S. Fan, J. Appl. Phys. 112, 024304 (2012).

${ }^{47}$ S. Basu and M. Francoeur, Appl. Phys. Lett. 98, 113106 (2011).

${ }^{48}$ P. Ben-Abdallah and S.-A. Biehs, Appl. Phys. Lett. 103, 191907 (2013).

${ }^{49}$ Y. Yang, S. Basu, and L. Wang, Appl. Phys. Lett. 103, 163101 (2013).

${ }^{50}$ M. F. Modest, Radiative Heat Transfer (Academic Press, 2013).

${ }^{51}$ M. Hu, P. Keblinski, and B. Li, Appl. Phys. Lett. 92, 211908 (2008).

${ }^{52}$ G. S. Springer, Adv. Heat Transfer 7, 163 (1971).

${ }^{53}$ M. Kaviany, Heat Transfer Physics (Cambridge University Press, 2014).

${ }^{54}$ D. D. Do, Adsorption Analysis: Equilibria and Kinetics: (With CD Containing Computer Matlab Programs) (World Scientific, 1998), Vol. 2.

${ }^{55}$ C. Wang, J. Chen, J. Shiomi, and S. Maruyama, Int. J. Therm. Sci. 46, 1203 (2007).

${ }^{56}$ G. Hwang and M. Kaviany, J. Appl. Phys. 106, 24317 (2009).

${ }^{57}$ G. S. Hwang and M. Kaviany, J. Appl. Phys. 106, 024317 (2009).

${ }^{58}$ Z. Liang, W. Evans, and P. Keblinski, Phys. Rev. E 87, 022119 (2013).

${ }^{59}$ Z. Liang and P. Keblinski, Int. J. Heat Mass Transfer 78, 161 (2014).

${ }^{60}$ H. Heinz, R. A. Vaia, B. L. Farmer, and R. R. Naik, J. Phys. Chem. C 112, 17281 (2008). 